\title{
EXCITED STATES IN THE DYNAMIC JAHN-TELLER EFFECT
}

\author{
J.C. SLONCZEWSKI and V.L. MORUZZI \\ IBM Watson Research Center, Yorktown Heights, New York \\ (Received 5 January 1967 )
}

\begin{abstract}
A study is made of the dynamic Jahn-Teller effect for the case of a doubly degenerate electronic state coupled to one pair of degenerate vibrational coordinates. Wave functions are computed electronically for boundary conditions appropriate to a study of upper-branch motion in a crystal containing an impurity or other imperfection, in the limit of vanishing harmonic potential. Plots of upper-branch lifetime vs. energy show resonances which progressively gain strength with increasing angular momentum. The computed spectrum of resonant levels is like one previously estimated but with significant corrections for small angular momentum and large radial quantum number. The computed lifetimes are markedly smaller than those estimated previously.
\end{abstract}

\section{Introduction}

WHEN the interaction between electrons and nuclei is strong, the dynamic Jahn-Teller effect may sometimes be thought of as a kind of hindered internal rotation about an impurity center or other imperfection in a crystal. What rotates, however, is not a molecular configuration but a cloud of electron-charge whose symmetry is lower than the nominal symmetry of the surrounding nuclear configuration. In a time-dependent representation of this rotation, the electron wave function may be approximated by a linear combination of degenerate states with time-dependent coefficients. In the unusual case in which these degenerate states might be approximated by pure $p$-states, the deformation of the charge cloud with time is purely rotational. In more general cases the crystal potential affects the charge density so that the rotary motion is modulated by a pulsation. In any case, the electrostatic attraction of the electrons causes the nuclei near the center to oscillate in synchronism with the electronic rotation, thus providing the system with kinetic energy [1].

In certain cases, the dynamic Jahn-Teller effect is conveniently studied in an adiabatic representation whose basis is an orthogonal set constructed from the $n$ degenerate electronic states, with coefficients which depend on the nuclear coordinates $Q$. In this representation, the $Q$-dependent diagonal elements of the $n \times n$ effective Hamiltonian matrix represent an $n$ valued potential for adiabatic, hindered-rotational, motion of the nuclei. The off-diagonal elements generally involve nuclear momenta and describe non-adiabatic effects. Therefore, an $n$-component wave function is generally required to describe the state of the system accurately. Non-adiabatic effects are particularly important under the conditions studied in this paper because their magnitude is comparable to that of the adiabatic splittings. They are, therefore, treated accurately, while the hindering potential, which is of higher order, is neglected: 
If spin-orbit effects are excluded from consideration one may distinguish two cases of electronic orbital degeneracy, doublets and triplets, which have different qualitative properties. An example of the doublet case, which is amenable to the "hindered rotation" point of view, is that of a $\mathrm{Cu}^{2+}$ impurity in octahedral coordination, whose static properties were first discussed by van vleck [2] and elaborated by öpik and Pryce [3]. Its dynamic properties were first discussed by Moffitt and Liehr [4]. Subsequently Longuet-Higgins et al. [5], and Moffitt and Thorson [6] computed eigenstates and eigenvalues of the effective Hamiltonian (including non-adiabatic terms) for this case under the assumption that one pair of degenerate vibrational modes interacts with the electrons and that the "hindering" potential is negligible. Furthermore, effects of the hindering terms on motion described by the lower branch of the 2-valued potential have been studied $[4,7]$, particularly in connection with spin resonance spectra $[8-10]$,

The case (not studied here) of an orbital triplet, which interacts with vibrational doublets and triplets, is not easily characterized as a hindered rotation, and the theory proceeds along other lines $[11,12]$.

In an earlier publication one of us investigated the orbital-doublet case including the coupling to a large set of vibrational modes representing the spectrum of the host crystal. We concluded that the two-coordinate model is meaningful for small displacements of the nuclei even in a general crystal. This observation heightens interest in motion on the upper branch of the potential because in the adiabatic limit stable upper-branch states appear which are tightly bound to the origin of $Q$-space when the electron-nuclear (or Jahn-Teller) coupling is large. In the neighborhood of this origin the potential approaches a circular cone giving rise to energy eigenvalues which we roughly estimated previously [13], and which we now compute electronically. The stability of these highly excited states in the adiabatic limit arises from the fact that the frequency elements of motion exceed the highest frequency available in the vibrational spectrum of the crystal so that the energy of the center cannot be transmitted away by propagation through the crystal except by high-order processes. In fact, however, non-adiabatic corrections to the motion are considerable, especially for the lower-lying upper-branch states. The consequent transitions to the lower branch provide a ready channel for transmission of energy away from the imperfection because lower branch motion has a low frequency and a large amplitude so that it communicates easily with the lattice [13]. It is better, therefore, to regard upper branch states as "resonances" rather than energy levels,

Further interest in upper-branch resonances has been stimulated by recent work of Höchli, Müller, and Wysling on spin-relaxation of certain transition metal impurities having double orbital degeneracy in the ground state [14]. In several cases, they found that the spin-relaxa tion time is proportional to $e^{+\Delta / k T}$ where $\Delta$ is a positive constant whose value depends on the particular impurity and host crystal studied. In some of the systems studied $\Delta$ was of such a magnitude that the only possible excited electronic state one could associate with it was the excited state arising from the Jahn-Teller splitting. This observation led them to suggest that, whereas the $g$-factor observed was characteristic principally of lower-branch motion, the spinrelaxation occurred principally during the brief periods of time in which the system was thermally excited to an upper branch state (presumably the lowest). An early attempt to analyze this mechanism was hindered by the unavailability of solutions to the two-component Schroedinger equation for the dynamical Jahn-Teller effect with boundary conditions appropriate to a crystal [15].

The object of the present investigation is to compute the wave functions describing upper branch motion of the two-coordinate model, and to describe accurately the resonant levels and lifetimes. However, no attempt is made here to compute the spin-relaxation time. In Section 2 we derive the effective Hamiltonian in the adiabatic representation. In Section 3 we explain 
how a boundary condition on the wave function used in the computations of Longuet-Higgins et al. [5] and Moffitt and Thorson [6] must be modified in order to dispose of the coupling between the local coordinate and the spectrum of crystal vibrations. We also derive an expression for the effective lifetime of upper-branch motion. In Section 4 we transform the Schroedinger equation into a form suitable for numerical computation and establish a procedure for satisfying the boundary conditions. In Section 5 we describe the program employed and the results of the computation. Examples of wave functions are shown, plots of upper-branch lifetime vs. energy are given, and the results are compared with previous work. In Section 6 additional effects neglected in the computations are discussed.

\section{Wave Equation}

We begin by deriving from considerations of symmetry an effective Hamiltonian which describes the Jahn-Teller effect in a system of two-fold electron-orbital degeneracy interacting with one pair of degenerate vibrational coordinates. The symmetry method has been used by Liehr [16] to derive the form of vibronic interaction for a host of molecular symmetries and applied by one of us [13] to the case of $n$ pairs of degenerate vibrations.

We assume a system consisting of a molecule, or of an impurity center in a crystal. We will expand the Hamiltonian about an initial nuclear configuration assumed to have a minimum symmetry of one three-fold rotational axis. Our conclusions will then apply to certain molecular or crysial-field symmetries such as trigonal, hexagonal, cubic, and regular-tetrahedral which include at least one three-fold axis. In particular, our Hamiltonian is equivalent to those of Longuet-Higgins et al. [5], and Moffitt and Thorson [6] for an octahedral complex.

We assume that the fundamental Hamiltonian for the system of electrons and nuclei does not contain spin-dependent terms. We further assume the existence of a degenerate pair of localized electronic orbital eigenstates $X_{+}$and $X_{-}$under the condition of vanishing displacement of the nuclear coordinate from the initial configuration. These states are assumed to satisfy the relations

$$
R_{X_{ \pm}}=\exp ( \pm 2 \pi i / 3) X_{ \pm}
$$

and

$$
x_{ \pm}^{*}=x_{F}
$$

where $R$ represents the rotation of the coordinate system in a given sense through an angle of $2 \pi / 3$ about the assumed axis and where * means complex conjugate. It is well known that all eigenstates not belonging to the identity representation of $R$ may be arranged into degenerate pairs satisfying equations of the sort (1) and (2) and we assume that we have one such pair to deal with.

We assume further a pair of real symmetry-degenerate normal coordinates $Q_{1}$ and $Q_{2}$ describing displacement of the nuclei from their initial configuration. From these we form the complex combinations

$$
Q_{ \pm}=Q_{1} \pm i Q_{2}
$$

which satisfy relations

$$
R Q_{ \pm}=\exp ( \pm 2 \pi i / 3) Q_{ \pm}
$$




$$
Q_{ \pm}^{*}=Q_{F}
$$

analogous to (1) and (2). In application to a crystal imperfection, $Q_{1}$ and $Q_{2}$ are particular linear combinations of all the normal modes which couple to the electrons [13]. In order to make these assumptions concrete, consider the hypothetical plane molecule (or complex embedded in a host crystal) composed of four nuclei shown in Fig. 1 (a). The initial configuration has

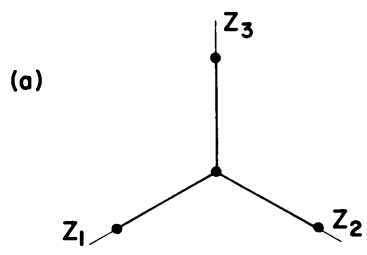

(b)

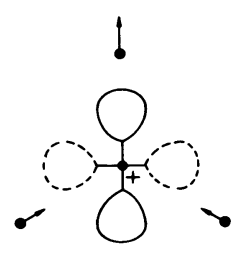

FIGURE 1

(a) Coordinate system of hypothetical $A B_{3}$ "molecule".

(b) Correlation of orbital orientation and nuclear position in the adiabatic representation. Arrowheads indicate the instantaneous nuclear configuration $\left(Q_{1}=0, Q_{2} \neq 0\right)$ corresponding to the pair of orthogonal p-orbitals indicated.

one nucleus at the center and three identical nuclei arranged symmetrically around the center. Assume that the molecule has, in addition to filled atomic shells and saturated chemical bonds, one electron tightly bound to the central nucleus in a two-dimensional $p$-state. In this case our basis functions may be written

$$
X_{ \pm}=e^{\mp i \theta} f\left(r_{e}\right) \text {, }
$$

where $r_{e}$ and $\theta$ are polar electron coordinates. Assume, very artificially, that the mass of the central nucleus is infinite and that only the radial displacements $Z_{1}, Z_{2}$, and $Z_{3}$ [shown in Fig. 1 (a)] of the three peripheral nuclei from this configuration are permitted. The degenerate vibrational coordinates in this case are

$$
\begin{aligned}
& Q_{1}=2^{-1 / 2}\left(Z_{1}-Z_{n}\right), \\
& \left.Q_{2}=6^{-1 / 2 / 2} Z_{3}-Z_{1}-Z_{2}\right) .
\end{aligned}
$$


We let $x_{e}$ represent the totality of several electronic coordinates in the general case and we approximate the electrostatic potential $v\left(x_{e}, Q_{1}, Q_{2}\right)$ with the linear form

$$
v=v_{+}\left(x_{e}\right) Q_{-}+v_{-}\left(x_{e}\right) Q_{+} \text {. }
$$

Here the coefficients satisfy the relation

$$
v_{+}^{*}=v_{-}
$$

because $v$ is real and $Q_{ \pm}$satisfy equation (5). Since $v$ is invariant with respect to rotation of the coordinate system, we have from equations (4) and (6),

$$
R v_{ \pm}=\exp ( \pm 2 \pi i / 3) v_{ \pm} \text {. }
$$

We proceed to calculate the matrix elements of $v$ in the basis $x_{ \pm}$. By applying the operation $R$, and taking the hermitean adjoints of the matrix elements $\left(X_{ \pm}, v_{ \pm} X_{ \pm}\right)$with all combinations of subscripts we find from the equations above, relations which reduce the representation of $v$ to the form

$$
\begin{aligned}
& \left(x_{+}, v x_{-}\right)=\left(x_{-}, v x_{+}\right)=A Q_{+} \\
& \left(x_{+}, v x_{+}\right)=\left(x_{-}, v x_{-}\right)=0 .
\end{aligned}
$$

Here the only independent constant is the complex Jahn-Teller coefficient

$$
A \equiv\left(\mathrm{x}_{+}, v_{-} \mathrm{x}_{-}\right)=\left(\mathrm{x}_{-}, v_{+} \mathrm{x}_{+}\right) *
$$

whose value is calculable only from explicit knowledge of the wave functions. The phase factor $e^{i \gamma}$ in this constant may be absorbed into $Q_{+}$by the transformation $Q_{+} \rightarrow Q_{+} e^{-i \gamma}$ which amounts to rotating orthogonal coordinate axes $Q_{1}$ and $Q_{2}$ through the angle $\gamma$. We assume that this transformation has been carried out so that $A$ in equation (9) is real and positive.

We take $P_{1}$ and $P_{2}$ to be nuclear momentum operators conjugate to $Q_{1}$ and $Q_{2}$ respectively and absorb the nuclear mass into the definitions of these coordinates in the usual way. Summing the harmonic vibrational energy and the .Tahn-Teller interaction (9) we obtain a model Hamiltonian expressed as a matrix in the space $\left[x_{+}, x_{-}\right]$by the form

$$
\mathscr{H}=\frac{1}{2}\left(P_{1}^{2}+P_{2}^{2}+\omega^{2} Q_{1}^{2}+\omega^{2} Q_{2}^{2}\right)\left(\begin{array}{ll}
1 & 0 \\
0 & 1
\end{array}\right)+A\left(\begin{array}{c}
0 \\
Q-0
\end{array} Q_{+}\right)
$$

where $\omega$ is formally the circular frequency of vibration for $Q_{1}$ and $Q_{2}$ in the absence of the Jahn-Teller interaction. In application to a crystal imperfection $\omega$ is not a natural frequency but is computable in principle from the vibrational spectrum of the orystal. $t$ in this representation the wave function for a stationary state has two components $\Psi_{+}\left(Q_{1}, Q_{2}\right)$ and $\Psi_{-}\left(Q_{1}, Q_{2}\right)$, which satisfy the eigenvalue equation

$$
\mathscr{H}\left(\begin{array}{c}
\psi_{+}^{+} \\
\psi_{-}
\end{array}\right)=E\left(\begin{array}{l}
\psi_{+}^{+} \\
\psi_{-}
\end{array}\right)
$$

and the normalization condition

+ our $\omega^{2}$ here is not the same as $\omega^{2}$ in [13] but is equal to $\sum_{i=1}^{n} \omega_{i}^{2} \lambda_{i n}^{2}$ in the notation of [13] 


$$
\int_{-\infty}^{\infty} \int_{-\infty}^{\infty}\left[\left|\psi_{+}\right|^{2}+\left|\psi_{-}\right|^{2}\right] d Q_{1} d Q_{2}=1 .
$$

We introduce real polar coordinates $r$ and $\phi$ through the relation

$$
Q_{ \pm}=r e^{ \pm i \varphi} .
$$

The interaction term in equation (11) is diagonalized and the rotational invariance of the system is brought out by the transformation

$$
\psi_{ \pm}=(2 r)-1 / 2 \mathrm{e}^{ \pm i \phi / 2}\left(\Phi_{1} \mp \Phi_{2}\right) \text {. }
$$

where $\Phi_{1}(r, \phi)$ and $\Phi_{2}(r, \phi)$ are the two components of the wave function in the new (adiabatic) representation. Upon transforming the differential form of equation (11) and (12) (with $\left.P_{i}=-i \hbar \partial / \partial Q_{i}\right)$ through relations (14) and (15) one finds equation (12) replaced by

$$
\hat{\mathscr{H}}\left(\begin{array}{c}
\Phi_{1} \\
\Phi_{2}
\end{array}\right)=E\left(\begin{array}{c}
\Phi_{1} \\
\Phi_{2}
\end{array}\right)
$$

where $\hat{\mathscr{H}}$ is the following physically interpretable Hamiltonian:

$$
\hat{\mathscr{H}}=\frac{J^{2}-\hbar \sigma_{1} J}{2 r^{2}}+\frac{P_{r}^{2}}{2}+A \sigma_{3} r+\frac{1}{2} \omega^{2} r^{2} .
$$

where $J=-i \hbar \partial / \partial \phi$ and $P_{r}=-i \hbar \partial / \partial r$ are momentum operators, and

$$
\sigma_{1} \equiv\left(\begin{array}{ll}
0 & 1 \\
1 & 0
\end{array}\right), \quad \sigma_{3} \equiv\left(\begin{array}{rr}
1 & 0 \\
0 & -1
\end{array}\right) .
$$

The first term in $\widehat{\mathscr{H}}$ is an effective centrifugal potential which includes a term (proportional to $\sigma_{1}$ ) which is an off-diagonal correction to the adiabatic approximation for nuclear motion in the presence of electrons. The remaining terms in equation (17) are respectively the radial kinetic energy, the (now-diagonal) Jahn-Teller interaction energy, and the harmonic potential.

The variables $r$ and $\phi$ in equation (16) are separated by the substitution

$$
\Phi_{1}=(2 \pi)^{-1 / 2} \mathrm{e}^{i m_{\Phi}} \alpha(r), \quad \Phi_{2}=(2 \pi)^{-1 / 2} \mathrm{e}^{i m} \Phi \beta(r)
$$

which yields a radial schroedinger equation

$$
\mathscr{H}^{\prime}\left(\begin{array}{l}
\alpha \\
\beta
\end{array}\right)=E\left(\begin{array}{l}
\alpha \\
\beta
\end{array}\right) \text {. }
$$

where $\mathscr{H}^{\prime}$ is given by equation (17) with $J$ replaced by $\hbar m$. Since the original nuclear state function $\left(\psi_{+}, \psi_{-}\right)$refers to the fixed electronic basis $\left(x_{+}, x_{-}\right)$, it satisfies the boundary condition

$$
\psi_{ \pm}(\phi+2 \pi)=\psi_{ \pm}(\phi)
$$

After the transformation (15), the nuclear state function $\left(\Phi_{1}, \Phi_{2}\right)$ refers to an electronic 
basis which is correlated with the nuclear coordinate $\varphi$. [In the case of our hypothetical plane molecule the new basis orbitals have the angular factors $\cos (\theta-\phi / 2)$ and $\sin (\theta-\phi / 2)$, and their correlation with nuclear positions is illustrated in Fig. 1 (b).] The presence of the factor $e^{ \pm i \phi / 2}$ in equation (15) converts equation (20) to the anti-periodicity condition

$$
\Phi_{1,2}(\phi+2 \pi)=-\Phi_{1,2}(\phi) \text {. }
$$

It follows that the allowed values of $m$ appearing in equation (18) are $\pm 1 / 2, \pm 3 / 2, \pm 5 / 2$, etc. Since all of the matrix elements of $\mathscr{H}^{\prime}$ are real, both $\alpha$ and $\beta$ may be taken real and the normalization (13) becomes

$$
\int_{0}^{\infty}\left(\alpha^{2}+\beta^{2}\right) d r=1 \text {. }
$$

\section{Boundary Conditions}

In works closely related to ours, Longuet-Higgins, et al. [5] and Moffitt and Thorson [6] computed eigenvalues and eigenstates of a Schroedinger equation equivalent to equation (19), although different in appearance. They used the boundary conditions (in our notation)

$$
\alpha(0)=\beta(0)=\alpha(\infty)=\beta(\infty)=0 \text {. }
$$

appropriate to a molecule in which one pair of degenerate vibrations couples to the electronic states. In application to an impurity or other solid state defect it is in general necessary to consider the coupling of the localized electronic state to a continuous distribution of vibrations as well as to any localized vibrations which might exist. In a previous publication we argued that the two-coordinate model still has some significance in this case for a region $r<r_{c}$ in which the harmonic force $(-\omega r)$ is small compared to the centrifugal $\left(\sim J^{2} / r^{3}\right)$ and Jahn-Teller $(\mp A)$ forces. In this case the coordinates $Q_{1}$ and $Q_{2}$ are some linear combinations of the interacting localized and non-localized normal coordinates which would exist in the absence of the Jahn-Teller coupling $[13,17]$.

It is clear that in general the bound $r_{c}$ must be considerably less than $r_{\mathrm{min}}$, the minimum of the lower branch of the effective potential (see Fig. 2), for, by definition, $r_{\min }$ is the point at which harmonic forces are sufficient to balance the sum of a positive Jahn-Teller and a positive centrifugal force. If $r_{c}$ is greater than the outer classical turning point $r_{i}$ on the upper branch of the potential (illustrated in Fig. 2) and if the effect of the non-diagonal centrifugal term $-\hbar \sigma_{1} J / 2 r^{2}$ is not too great, then there should exist well-defined resonant oscillations about the point $r=r_{0}$, with the electron system occupying the upper state.

Earlier we concluded that a necessary condition for some of these resonances to exist is the inequality

$$
E_{s} \equiv \frac{A^{2}}{\omega^{2}} \gg h \omega
$$

where $E_{s}$ is the classical static stabilization energy evaluated as the magnitude of the lower branch of the potential with $J=0$ and $r=r_{\mathrm{min}}$ [13]. We also gave rough estimates of upperbranch levels, based on a harmonic approximation of the upper-branch potential, and of lifetimes based on crude perturbation theory. A proper calculation requires solution of the two- 
component Schroedinger equation (19), which we do here numerically.

In order to study the lifetimes of the resonant states we should in principle solve a timedependent wave equation. We avoid this by considering an artificial resonant reflection process which permits us to use stationary states. We assume equal steady rates of probability flow in the positive and negative radial directions on the lower branch at some large value of $r$. We then calculate the average time an incoming "particle" remains on the upper branch before it is re-emitted. We assume that there is a range of $r$ less than $r_{c}$ in which the WKB approximation on

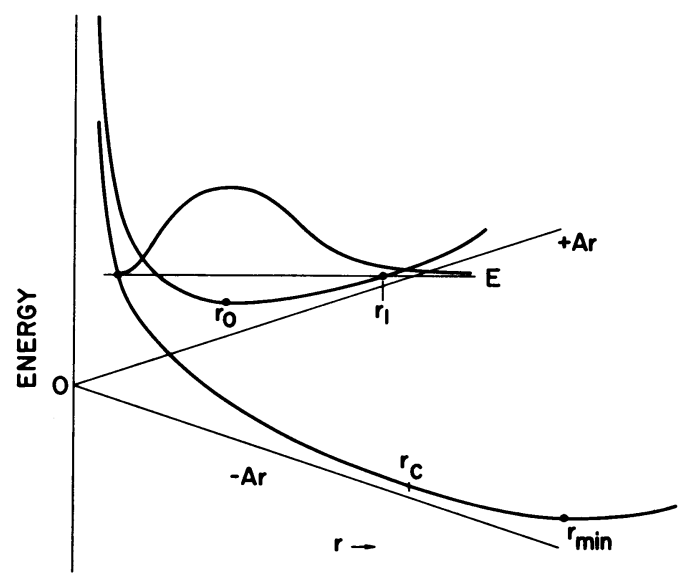

FIGURE 2

Double-valued "radial" potential in the adiabatic limit of the dynamical

Jahn-Teller effect. The Jahn-Teller splitting is indicated by the

straight lines and the total potential including Jahn-Teller, centrifugal, and harmonic potentials is shown by the two upward concave curves.

The two branches of the potential correspond to the two electronic states.

Here $r$ is the radius in a two-dimensional configuration space.

the lower branch is valid. Since the Hamiltonian is real we may write in such a region a solution of equation (19) in the form

$$
\alpha=0, \beta_{w}(E, r)=B(E) k^{-1 / 2}(r) \sin \int_{\hat{r}(E)}^{r} k\left(r^{\prime}\right) d r^{\circ} .
$$

where $k(r)$ is the classical momentum

$$
k(r)=\hbar^{-1}\left\{2\left[E-\hbar^{2} m^{2} / 2 r^{2}+A r-\frac{1}{2} \omega^{2} r^{2}\right]\right\}^{1 / 2} \text {. }
$$

and where $\hat{r}$ is a parameter which depends on $E$ and can be determined in principle from the exact solution of equation (19) with the boundary conditions $\alpha(0)=\beta(0)=\alpha(\infty)=0$. The coefficient $B$ is a normalizing constant which depends on $E$.

Equation (24) represents a standing wave which superposes equal and opposite particle fluxes of amount $\hbar B^{2} / 4$ "particles" per unit time. The average time $T$ which the system spends on the upper branch during each encounter is 


$$
T=\frac{4 \int_{0}^{\infty} \alpha^{2} d r}{\hbar B^{2}}
$$

where $\alpha$ is taken from the exact solution of the two-component Schroedinger equation and the constant $B$ is determined by setting equation (24) equal to the exact $\beta$ in the neighborhood of an appropriate value $\bar{r}$ of $r$. The fact that equation (24) has two parameters $B$ and $\hat{r}$ to be determined suggests calculating $B$ from $\beta(\bar{r})$ and $\beta^{\prime}(\bar{r})$ where 'means derivative. By differentiating equation (24) once and combining the resulting equation with equation (24) to eliminate trigonometric functions one finds

$$
B^{2}=k \beta^{2}+k^{-1}\left[\beta^{\prime}+\frac{k^{\prime}}{2 k} \beta\right]^{2} .
$$

where we have replaced $\beta_{w}$ by the exact $\beta$.

\section{Method of Computation}

For purposes of numerical computation it is convenient to write the Schroedinger equation in dimensionless units. We let $\lambda$ and $x$ be the energy and distance, respectively, in the units found convenient in our earlier paper:

$$
E=(\hbar A)^{2 / 3} \lambda, \quad r=\left(\hbar^{2 / 3} / A^{1 / 3}\right) x
$$

Also, we will confine ourselves to the special case $\omega=0$, which is permissible because of the absence of the boundary condition on $\beta(\infty)$. Then in these units the minimum of the upper potential branch is simply $x_{0}=m^{2 / 3}$. The radial Schroedinger equation (19) now becomes

$$
\begin{aligned}
& d^{2} \alpha / d x^{2}+\left(2 \lambda-m^{2} / x^{2}-2 x\right) \alpha+m \beta / x^{2}=0 \\
& d^{2} \beta / d x^{2}+\left(2 \lambda-m^{2} / x^{2}+2 x\right) \beta+m \alpha / x^{2}=0
\end{aligned}
$$

for $x>0$. Since these equations are invariant under the transformation $\alpha \rightarrow+\alpha, \beta \rightarrow-\beta$. $m \rightarrow-m$, we see that all levels are two-fold degenerate. In the remainder of this section we take $m$ to be positive.

The usual sort of analysis of equations (28) for small $x$, in conjunction with the boundary condition $\alpha(0)=\beta(0)=0$, shows the limiting behavior to be given by

$$
\begin{aligned}
& \alpha=a x^{m}+b x^{m+1}+\ldots . \\
& \beta=a x^{m}-b x^{m+1}+\ldots
\end{aligned}
$$

where $a$ and $b$ are constants of integration. The ratio $a / b$ is determined in principle by the remaining condition $\alpha(\infty)=0$. 
In a point-by-point numerical integration it is convenient to begin at the origin with finite values. Therefore, in view of equation (29), we make the transformation

$$
\alpha=x^{m} u, \quad \beta=x^{m} v
$$

whose substitution in equation (28) yields the equations

$$
\begin{aligned}
& x^{2} d^{2} u / d x^{2}+2 m x d u / d x+\left[2(\lambda-x) x^{2}-m\right] u+m v=0, \\
& x^{2} d^{2} v / d x^{2}+2 m x d v / d x+\left[2(\lambda+x) x^{2}-m\right] v+m u=0,
\end{aligned}
$$

for $x>0$. Since a numerical integration can only proceed by steps from one end of the interval to the other we must specify four initial conditions. From equations (29) and (30), these conditions have the form

$$
u(0)=v(0)=a, \quad d u(0) / d x=-d v(0) / d x=b \text {, }
$$

where $a$ and $b$ are the coefficients introduced in equation (29), and are arbitrary for the moment. Also, limiting forms of equations (31)

$$
d^{2} u(0) / d x^{2}=\frac{-\lambda a}{m+1 / 2}=d^{2} v(0) / d x^{2} \text {, }
$$

must be substituted into the integration routine at $x=0$.

In principle, the boundary condition $\alpha(\infty)=0$ may be satisfied by superposing any two solutions having different ratios $a / b$ with appropriate coefficients. In computational practice, a finite boundary point is required and we iterate an approach to the boundary condition as follows. First, we determine a limiting form for $\alpha(x)$ by noting that, for a narrow range of very large $x$ values, the coefficients of the differential equations (28) may be considered constant. Since $x$ is in a classically forbidded region of the upper branch, $\alpha$ is small and we neglect the term $m \alpha / x^{2}$ in equation (28b). We find that $\beta$ is proportional to $\cos (\kappa x-\gamma)$ where $k$ is given by

$$
k^{2}=2 \lambda-m^{2} / x^{2}+2 x
$$

and $\gamma$ is a constant. Substituting this form of $\beta$ into equations (28a) and (30) we find that for large $x$, a solution satisfying the condition $u(\infty)=0$ has the asymptotic form

$$
u=\frac{m v}{4 x^{3}}(x \rightarrow \infty)
$$

This relation represents a "forced" oscillation in a classically inaccessible region of the upper potential by the oscillation on the lower branch.

If we arbitrarily set $a=1$, the relation (34) leads us to select the value of $b$ in the $n^{\prime}$ th attempt at integration according to the algorithm

$$
b_{n}=\frac{\left[4 \bar{x}^{3} u_{n-1}(\bar{x})-m v_{n-1}(\bar{x})\right] b_{n-2}-\left[4 \bar{x}^{3} u_{n-2}(\bar{x})-m v_{n-2}(\bar{x})\right] b_{n-1}}{4 \bar{x}^{3}\left[u_{n-1}(\bar{x})-u_{n-2}(\bar{x})\right]-m\left[v_{n-1}(\bar{x})-v_{n-2}(\bar{x})\right]}
$$


where $u_{n}, v_{n}$ are the functions $u$ and $v$ integrated using the value $b=b_{n}$, and $\bar{x}$ is a selected boundary point. One may verify that if $b_{n-1} \neq b_{n-2}$, then the solution with $b$ given by equation (35) satisfies equation (34) at $x=\bar{x}$ exactly. In a numerical integration it is better to use this relation as a basis for iteration because the exponentially growing character of the $\alpha-$ solution integrated over a wide range of $x$ introduces computational errors.

\section{Machine Computations and Results}

In the computer program for the numerical integration of equations (31) and (32), we use Gills' variation of the method of Kutta in which the error in each step is of the order of $h^{5}$, where $h$ is the interval $[18,19]$. For given values of angular momentum $(m)$ and energy $(\lambda)$, we

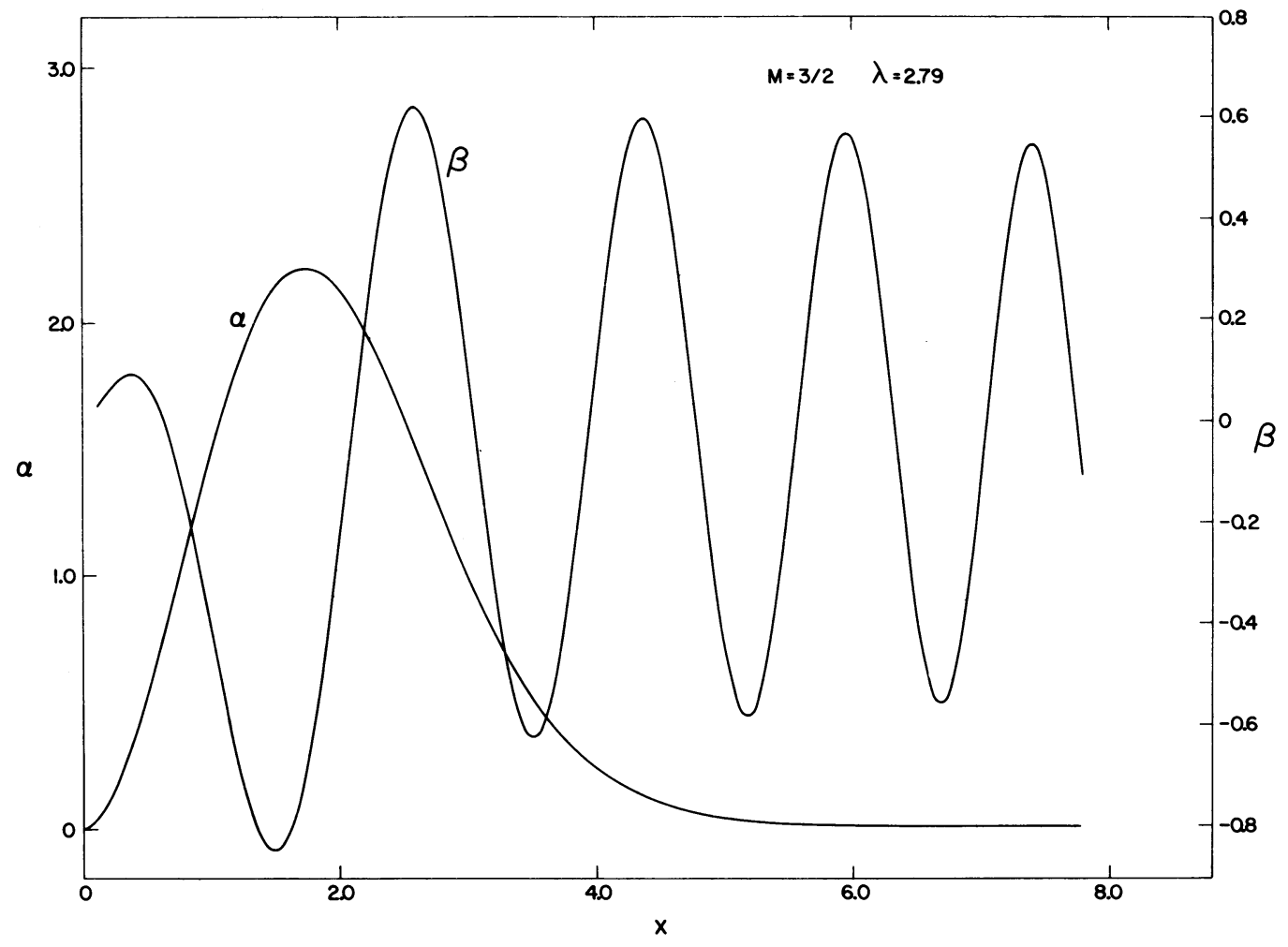

FIGURE 3

Numerical solution of the radial schroedinger equation for angular momentum $m=3 / 2$ and energy $\lambda=2.79$.

The amplitude $\alpha$ (upper electronic state) and $\beta$ (lower state) are plotted vs. the reduced configurational radius $x$.

perform a step-by-step integration over a range of values of $x$. For most of the computations, the integration is from $x=0$ to $x=\bar{x} \approx \lambda+5.0$ in intervals corresponding to $1 / 200$ th of the range. Successive integrations with $b_{n}$ given by equation (35), beginning with $n=3$, are then performed with the initial values $b_{1}$ and $b_{2}$ chosen at random (the computations converge rapidly for any reasonable $b_{1}$ and $b_{2}$ values). The iteration scheme was considered satisfied when 
successive integrations gave

$$
\left|b_{n}-b_{n-1}\right| \leqslant 0.2 \times 10^{-7}
$$

More stringent conditions on the evaluation of the integration constants produced insignificant improvements and tended toward long computation times.

In Fig. 3, we show computed wave functions for the case $m=3 / 2, \lambda=2$. 79. On the lower branch, the kinetic energy is positive for all, except very smail, values of $x$ and, therefore, $\beta$ has the expected oscillatory behavior. The upper branch has a classically allowed region lying between two forbidden regions. The behavior in the allowed region about the minimum in the upper branch depends on the proximity of $\lambda$ to a resonance. Figure 3 , which shows $\alpha$ with one large ant node, illustrates the solution for a first resonance. In Fig. 4, we show the changing character of $\alpha / B$,

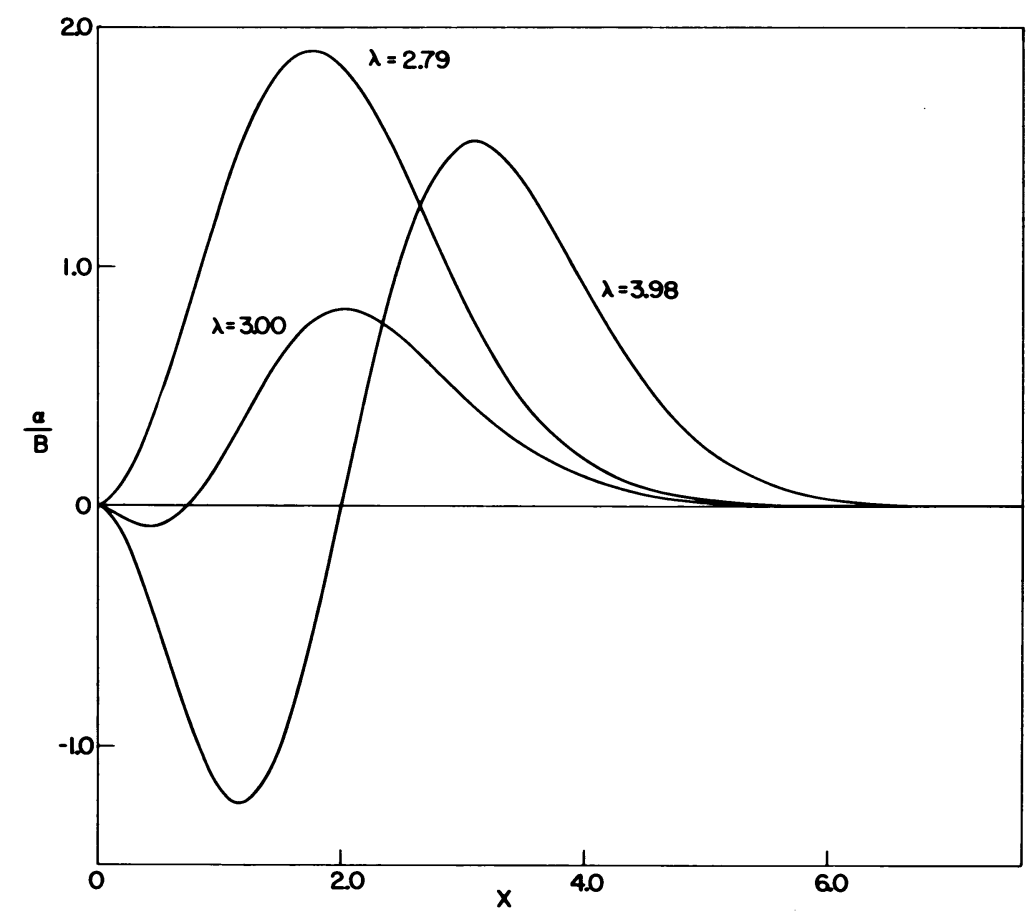

FIGURE 4

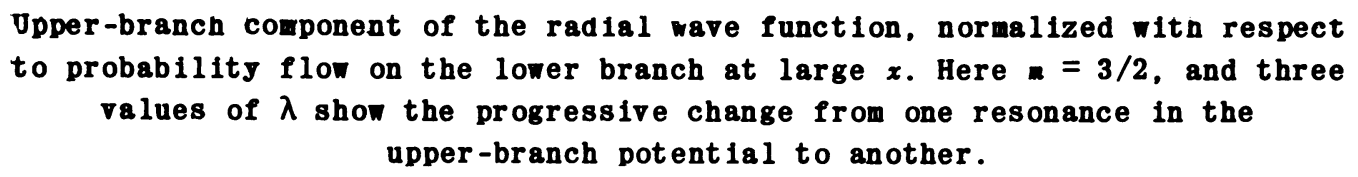

where $B$ is the normalizing factor (23), as we increase $\lambda$ from a first to a second resonance. The two resonant cases, $\lambda=2.79$ and $\lambda=3.89$, show one and two large antinodes respectively. The intermediate case for $\lambda=3.0$ shows the second antinode in an early state of growth near the origin. In a gross sense, $\alpha$ has an exponentially decaying behavior in the forbidden regions. Superimposed on the decay in the outer region, however, is the "forced" oscillatory behavior described by equation (34). These forced oscillations, which are too small to be perceived in 
Figs. 3 and 4, but are more pronounced for larger $|m|$, are displayed in Fig. 5 for the (offresonance) case $m=11 / 2, \lambda=5.6$.

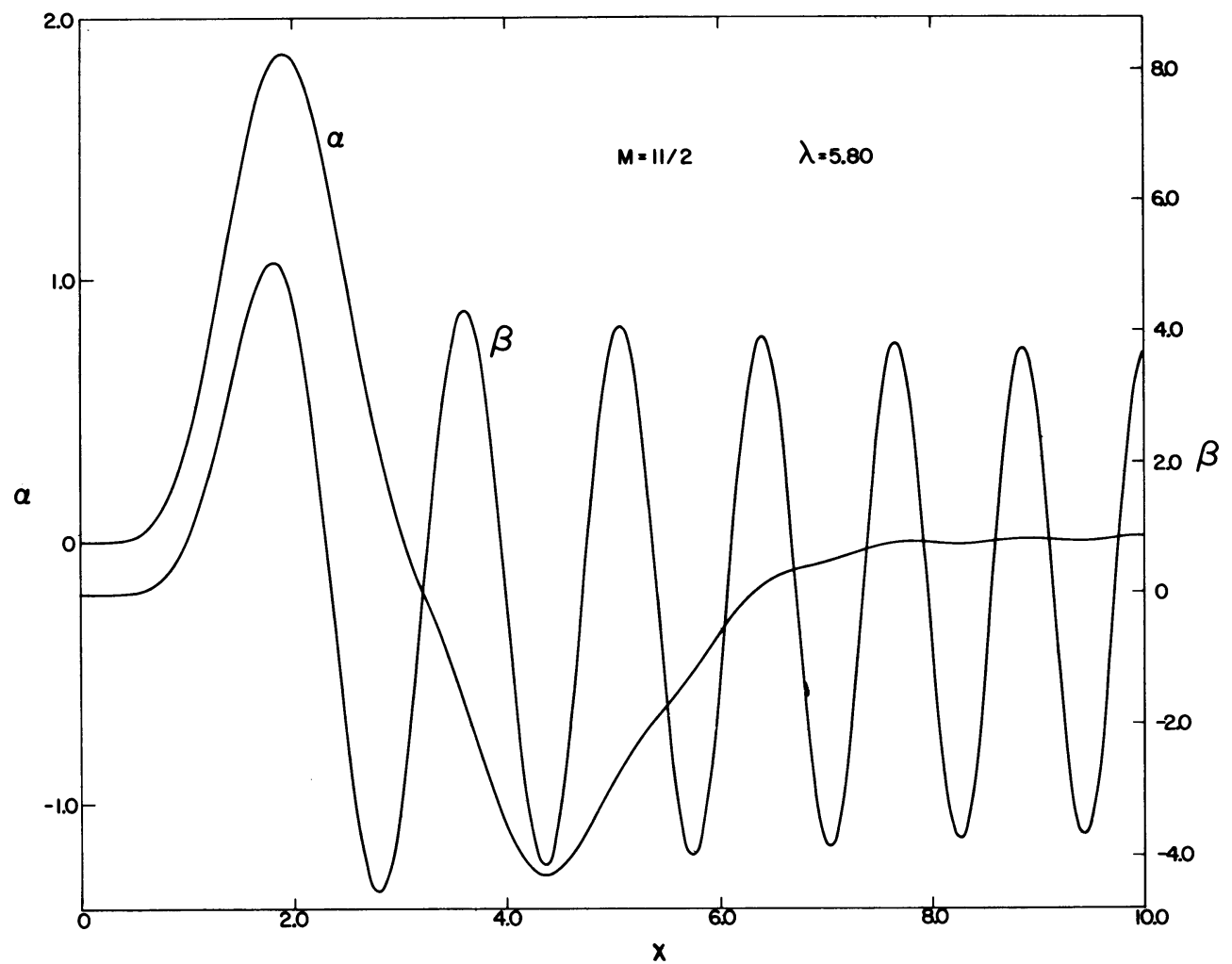

FIGURE 5

Forced $\alpha$-component oscillations for $m=11 / 2$ and $\lambda=5.6$.

They appear as small irregularities in phase with $\beta$-oscillations.

The resonant character of the system is displayed comprehensively by the plots of reduced lifetime $T$ vs. energy in Fig. 6. Here $T$ is related to $T$ of equation (25) by

$$
T=4 \hbar^{1 / 3} T / A^{2 / 3} \text {. }
$$

These units are such that $T$ is on the order of the mean number of upper-branch oscillations executed by the system after being directed initially along the lower branch from infinity toward the origin, before it is ejected again via the lower branch.

Approximate resonant levels $\lambda_{m s}$ and lifetime $\tau_{m s}$ obtained from peaks of curves such as those of Fig. 6 are shown in Table 1, where $s$ is the number of radial nodes in the classically allowed region of the upper branch. We note that the resonance is broad and weak for smal 1 values of $m$. This result arises from the fact that the ratio of off-diagonal to diagonal matrix elements of the centrifugal potential operator is $|m|^{-1}$ so that only for large $|m|$ can the branch coupling be considered small. The values of $\lambda_{m s}$ computed here agree with estimates based on a harmonic approximation to the upper-branch potential [13] except when $|m|$ is small and $s$ 


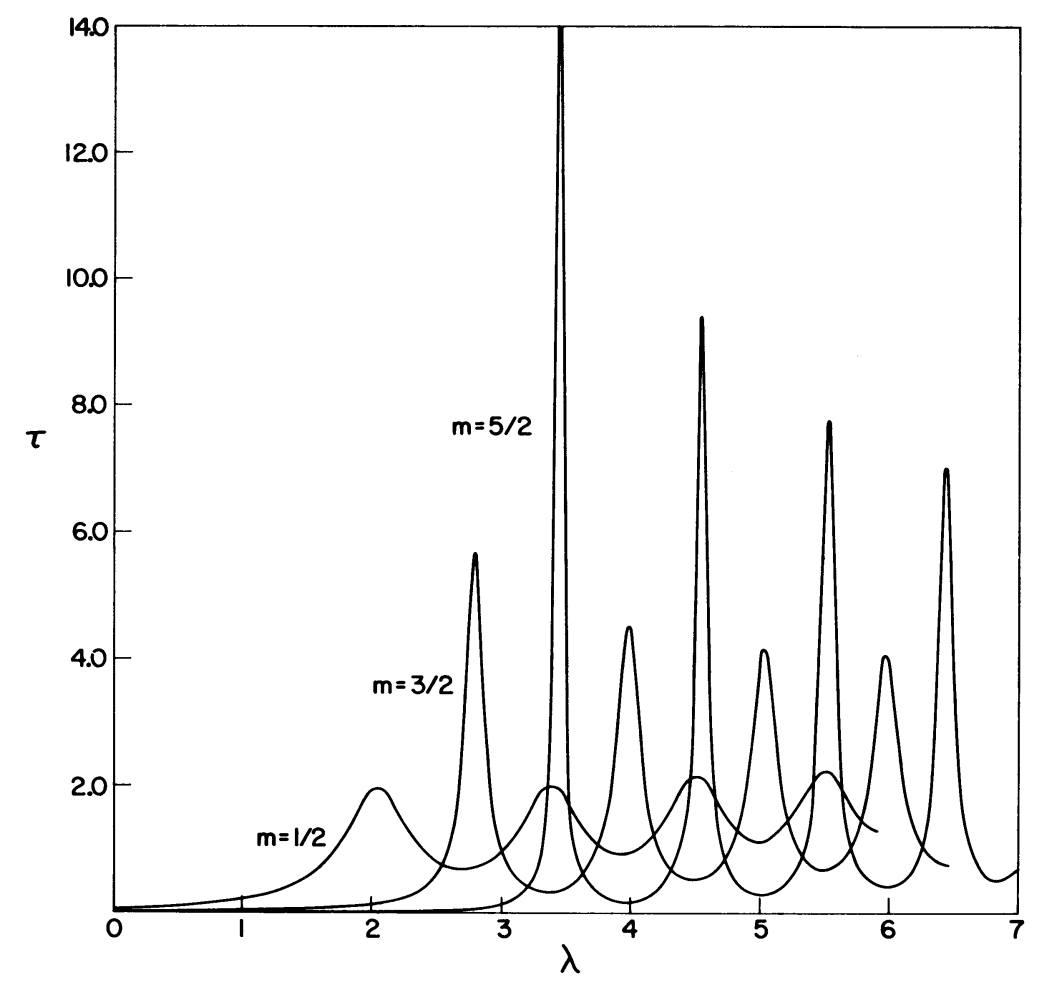

FIGURE 6

Reduced lifetime-T of the excited electronic state vs. reduced energy for $|m|=1 / 2,3 / 2,5 / 2$.

The peaks represent resonances in the upper-branch potentials.

is at the same time large, because in these cases the upper-branch potential deviates considerably from harmonic. The computed lifetimes are considerably smaller than those estimated earlier and show the inadequacy of the simple approach which had been used [13].

Finally, we should compare our results with those of Longuet-Higgins et al. [5] and Moffit and Thorson [6]. Since both of these works used a boundary condition equivalent in our notation to $\beta(\infty)=0$ and obtained energy eignevalues rather than lifetimes, the comparison can only be indirect and qualitative. To show evidence of resonant behavior in a continuous set of eigenvalues we need to display peaks in the density of states. When the spectrum is discrete, the analogue to the density of states is simply $\left(E_{i+1}-E_{i}\right)^{-1}$ where $E_{i}$ is the $i$ th energy eigenvalue in ascending order.

In the units of Longuet-Higgins the restoring force constant is unity and the Jahn-Teller coupling is measured by a quantity $k^{2}$. Our limit of vanishing harmonic potential corresponds to the limit $k^{2} \rightarrow \infty$ in their notation. Therefore, the best comparison to make is with their results for their largest value, $k^{2}=30$. (We do not attempt a separate comparison with Moffit and Thorson because all their results are for $k^{2} \leqslant 3$ and they agree with Longuet-Higgins, et al. in this range.) Noting that our $m$ is $l$ in their notation we take the values of $E_{i}$ for $k^{2}=30$, $l=1 / 2,3 / 2$ from their Table 4 [5]. In Fig. 7 we plot $\left(E_{i+1}-E_{i}\right)^{-1}$ vs. the mean energy 


\section{TABLE 1}

Values of reduced resonant levels $\lambda_{m s}$ and 11 fetimes $\tau_{m s}$ taken from peaks in Fig. 6 and similar curves, where $m$ and $s$ are rotational and radial quantum numbers, respectively.

\begin{tabular}{|c|c|c|c|}
\hline$|m|$ & $s$ & $\lambda_{m s}$ & $\tau_{m s}$ \\
\hline \multirow{4}{*}{$\frac{1}{2}$} & 0 & 2.05 & 1.95 \\
\hline & 1 & 3.38 & 2.02 \\
\hline & 2 & 4.50 & 2. 15 \\
\hline & 3 & 5.49 & 2.26 \\
\hline \multirow{4}{*}{$\frac{3}{2}$} & 0 & 2.78 & 5.8 \\
\hline & 1 & 3.98 & 4.6 \\
\hline & 2 & 5.03 & 4.2 \\
\hline & 3 & 5.97 & 4.0 \\
\hline \multirow{4}{*}{$\frac{5}{2}$} & 0 & 3.45 & 14.2 \\
\hline & 1 & 4.54 & 9.4 \\
\hline & 2 & 5.53 & 7.8 \\
\hline & 3 & 6.43 & 7.0 \\
\hline \multirow{4}{*}{$\frac{7}{2}$} & 0 & 4.06 & 29.1 \\
\hline & 1 & 5.08 & 17.3 \\
\hline & 2 & 6.02 & 14.2 \\
\hline & 3 & 6.89 & 11.5 \\
\hline \multirow{4}{*}{$\frac{9}{2}$} & 0 & 4.64 & 78.7 \\
\hline & 1 & 5.60 & 36.8 \\
\hline & 2 & 6.50 & 23.4 \\
\hline & 3 & 7.33 & 20.0 \\
\hline \multirow{4}{*}{$\frac{11}{2}$} & 0 & 5.18 & 174.0 \\
\hline & 1 & 6.10 & 67.0 \\
\hline & 2 & 6.95 & 46.0 \\
\hline & 3 & 7.76 & 35.0 \\
\hline \multirow{4}{*}{$\frac{13}{2}$} & 0 & 5.70 & 213.0 \\
\hline & 1 & 6.57 & 110.0 \\
\hline & 2 & 7.40 & 87.0 \\
\hline & 3 & 8.18 & 57.0 \\
\hline
\end{tabular}


$\left(E_{i}+E_{i+1}\right) / 2(30)^{1 / 3}$ where the factor (30) $1 / 3$ converts to our energy units. One sees resonant peaks in both instances $m=1 / 2$, and $3 / 2$, at about the same positions as in our Fig. 6 and also that larger $\left.\right|_{\mathrm{m}} \mid$ gives rise to sharper peaks, indicating that our results are consitent with theirs.

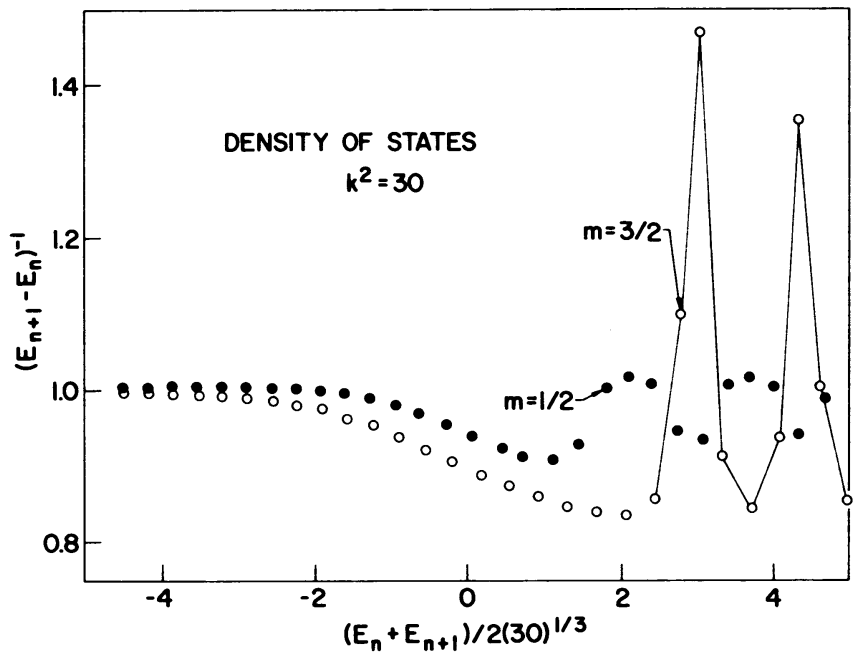

\section{FIGURE 7}

Plot of the inverse level separations v8. mean energy, taken fron Longuet-

Higgins et al. [5], for Jahn-Teller coupling coefficient $k^{2}=30$, showing resemblance to our lifetimes plotted in Fig. 6.

\section{Higher-Order Effects}

We must remark that $m$ is a good quantum number for the system under study only within the 1imitations of approximations made in deriving the Hamiltonian (17). Higher-order terms in the Hamiltonian compatible with the three-fold axis will couple resonant states with different $|m|$ and split certain states with the same $|m|$. In the case of an octahedrally coordinated complex, Öpik and Pryce [3] have shown that Born repulsion of the ionic cores gives rise to a diagonal term in the potential proportional to $r^{3} \cos 3 \theta$.

The effect of this term is evidently to couple states according to the selection rule $\Delta m= \pm 3$. Thus, in first order approximation, levels with $|m|=3 / 2$ will be split. Moreover, in still higher-order approximation, resonant levels satisfying $|m|=3 p / 2$, where $p$ is an integer, will also be split, but presumably more weakly with increasing values of $p$. Also, we expect that pairs of states satisfying $\Delta_{m}= \pm 3$, but which are nearly degenerate because of differing $s$ values, (e.g. the states $\lambda_{ \pm 1 / 2, y}=3.38$ and $\lambda_{F 5 / 2,0}=3.45$ in Table 1) will be shifted in first order. Additional higher-order terms discussed by Liehr and Ballhausen are non-diagonal in our representation, and should decrease lifetimes as well as split levels.

It is also apparent that levels with high $|m|$ and $s$ values involve excursions to large values of $r$ where we have remarked the two-coordinate model must in general be modified by coupling to the vibrational continuum of the solid. Within the framework of the two-coordinate model this 
coupling should become manifest as additional level broadening and decrease of lifetime, becoming more pronounced with increasing $|m|$ and $s$ so that for sufficiently large values the resonant levels lose significance. Although the harmonic term $(1 / 2) \omega^{2} r^{2}$, neglected in our computation, will produce significant level shifts in these circumstances, it will not alter the qualitative nature of the spectrum.

\section{Acknowledgement}

We are deeply indebted to Dr. U. Höchli for preliminary computations performed at the IBM Zurich Laboratory and for private discussion of his work.

\section{References}

1. For reviews of the dynamic Jahn-Teller effect, see H.C. LONGUET-HIGGINS, Adv. Spectrosc. 2, 429 (1961); and N.S. HAM, Spectrochim. Acta 18, 775 (1962).

Reference 11, below, also contains many references to recent literature.

2. J.H. VAN VLECK, J. Chem. Phys. 7, 72 (1939).

3. U. ÖPIK and M.H.L. PRYCE, Proc. R. Soc. A238, 425 (1957).

4. W. MOFFITT and A.D. LIEHR, Phys. Rev. 106, 1195 (1957).

5. H. C. LONGUET-Higgins, U. ÖPIK, M.H.L. PRYCE and R.A. SACK, Proc. R. Soc. A244, 1 (1958).

6. W. MOFFITT and W.R. THORSON, Calcul des Fonctions d'Onde Moleculaire (ed. R. Daudel), Rec. mem. C.N.R.S., Paris (1958).

7. I. B. BERSUKER, Zh, eksp. teor. Fiz. (U.S.S.R.) 43, 1315 (1962) [English translation: Soviet Phys. JETP 16, 933 (1963)].

8. V.I. AVVAKUMOV, Zh. eksp. teor. Fiz. 37, 1017 (1959) [English translation:Soviet Phys. JETP 10, $723(196: 0)]$.

9. I. B. BERSUKER, Zh. eksp. teor. Fiz. 44, 1239 (1963) [English translation: Soviet Phys. JETP 17. $836(1963)]$.

10. M.C.M. O'BRIEN, Proceedings of the First International Conference on Paramagnetic Resonance, p. 322. Academic Press, New York (1963); Proc. R. Soc. A281, 323 (1964).

11. F.S. HAM, Phys. Rev. 138, A1727 (1965).

12. M. CANER and R. ENGLman, J. Chem. Phys. 44, 4054 (1966).

13. J.C. SLONCZEWSKI, Phys, Rev, 131, 1596 (1963).

14. U. HÖCHLI and K.A. MÜLlER, Phys. Rev. Let t. 12, 730 (1964); U. HÖCHLI, K.A. MÜLLER and P. WYSLING, Phys. Lett. 15, 5 (1965); P. WYSLING, K. A. MÜLLER and U. Höchli, Helv. phys. Acta 37, 629 (1964) and 38, 358 (1965); K. A. MULLER, International Conference on Magnetic 
Resonance and Relaxation - XIVth Colloque Ampere, Ljubljana, Sept. 6-11 (1966).

15. J.C. SLONCZEWSKI, Helv. phys. Acta 38, 359 (1965) (abstract).

16. A. D. LIEHR, J. Phys. Chem. 67, 389 (1963).

17. The use of similar linear combinations. called "interaction modes", is advocated bv Y. TOYOZAWA and M. INOUE, J. phys. Soc. Japan 21, 1663 (1966).

18. S. GILL, Proc. Camb. phil. Soc. 47, 96 (1951).

gram was adapted from SHARE SDA 3240. 